\title{
HỆ TỌA ĐỘ KHÔNG GIAN QUỐC GIA GẮN LIỀN VỚI MẢNG KIỂN TẠO - XU THẾ CỦA VIỆC XÂY DỬNG HỆ TỌA ĐỘ QUỐC GIA HIỆN ĐẠI TRÊN THẾ GIỚI
}

\author{
HÀ MINH HÒA \\ Viện Khoa học Đo đạc và Bản đồ
}

\section{Tóm tắt:}

Trong quá trìn xây dụng hệ tọa độ không gian quốc gia, một vấn để được quan tâm là sụ ổn định của hệ độ cao quốc gia trong bài toán xác định mô hình quasigeod độ chính xác cao. Do mạng luới độ cao hạng I, II đã khai thác nhiều năm, các mốc độ cao bị xê dịch do các hoạt động kinh tế - xã hội và kiến tạo, việc đo lặp lại mạng luoới này rất tốn kém về kinh phí và thời gian, nên nhiều nước đã chỉ lự chọn các mốc độ cao ổn định trên thực địa để làm khớp với mô hình quasigeoid được xây dụng nhờ dũ liệu trọng lục. Ở Việt nam, mô hìn quasigeoid VIGAC2017 cũng được xây dưng theo cách tiếp cận này. Ngoài ra, hiện nay hệ tọa độ không gian quốc gia là hệ tọa độ gắn liền với mảng kiến tạo. Bài báo khoa học này sẽ xem xét các vấn đề nêu trên.

\section{1. Đặt vấn đề}

Trong lĩnh vực Đo đạc và Bản đồ, theo cách tiếp cận truyền thống, các cơ sở khống chế tọa độ mặt bằng và độ cao quốc gia là các điểm thiên văn - trắc địa hạng $\mathrm{I}$, II và các điểm độ cao hạng $\mathrm{I}$, II nhà nước một cách tương ứng. Đặc điểm của các điểm khống chế tọa độ mặt bằng và các điểm khống chế độ cao nhà nước là chúng được xây dựng riêng rẽ, không gắn kết với nhau, ngoài ra các điểm khống chế độ cao nhà nước thuộc mạng lưới độ cao hạng I, II được xây dựng trong một giai đoạn rất dài và rất tốn kém chi phí của Nhà nước do vừa phải đảm bảo sự thông hướng giữa hai điểm kề nhau phụ thuộc vào đặc điểm của địa hình, vừa phải đo đi - đo về, riêng các điểm độ cao hạng I còn đòi hỏi phải đo thêm đường trái - đường phải. Không những thế, các điểm khống chế độ cao nhà nước thường không ổn định do sự dịch chuyển của vỏ trái đất, đặc biệt ở các khu vực không ổn định về mặt địa chất.

Đối với các điểm không chế mặt bằng thuộc mạng lưới thiên văn - trắc địa hạng I, II ở các khu vực rừng núi cao đòi hỏi phải dựng các tiêu cao đảm bảo sự thông hướng giữa các điểm. Ngoài ra tại các khu vực rừng núi cao hiểm trở không thể tiến hành đo nối độ cao nhà nước vào các điểm thiến văn - trắc địa, nên độ cao nhà nước thường được xác định bằng phương pháp thủy chuẩn lượng giác đảm bảo độ chính xác $\pm 1 \mathrm{~m}$. Khi xử lý toán học mạng lưới thiên văn - trắc địa hạng I, II, chúng ta thường xác định các độ cao quasigeoid của các điểm bằng các phương pháp thủy chuẩn - thiên văn hoặc thủy chuẩn thiên văn - trọng lực. Điều này đảm bảo các độ cao trắc địa của các thiên văn - trắc địa hạng I, II luôn nhỏ hơn $\pm 2 \mathrm{~m}$. Với độ chính xác nên trên của các độ cao trắc địa của các thiên văn - trắc địa hạng I, II, hệ tọa độ đươc xây dựng dựa trên các thiên văn - trắc địa hạng I, II chỉ là hệ tọa độ $2 \mathrm{D}$ và các độ cao trắc địa của các thiên văn - trắc địa hạng $\mathrm{I}$, II với độ chính xác nhỏ hơn $\pm 2 \mathrm{~m}$ chỉ đủ để giải quyết bài toán quy chiếu các phương vị Laplace, các cạnh đáy, các hướng đo và các cạnh đo trong các mạng lưới đường truyền hạng I, II từ mặt vật lý trái đất lên mặt ellipsoid quy chiếu trong quá trình tính toán khái lược. 
Một đặc điểm nữa khi tính toán khái lược và bình sai mạng lưới thiên văn - trắc địa hạng I, II hoặc mạng lưới độ cao hạng I, II là việc truyền các tọa độ phẳng hoặc độ cao xuất phát từ một điểm gốc. Do sự tích lũy của các sai số làm tròn, nên các điểm càng xa điểm gốc càng chứa các sai số càng lớn trong các tọa độ phẳng hoặc độ cao. Do đó sai số vị trí tương hỗ mặt bằng giữa hai điểm thiên văn - trắc địa hạng I, II thường nằm ở mức $\pm 2-4 \mathrm{~cm}$, còn sai số trung phương lớn nhất của độ cao hạng I nằm ở mức $\pm 6,4 \mathrm{~cm}$, sai số trung phương lớn nhất của độ cao hạng $\mathrm{II}- \pm 7,5 \mathrm{~cm}$ (Hà Minh Hòa, 2014b).

Với sự xuất hiện của công nghệ GNSS vào đầu thập niên 1990, khi sử lý dữ liệu GNSS trong ITRF với việc sử dụng các dịch vụ của tổ chức IGS, độ chính xác vị trí mặt bằng tương hỗ $M_{x y}$ giữa hai điểm và độ chính xác của độ cao trắc địa $\hbar_{\overline{p y}}$ của điểm GNSS đạt được ở các mức (Hà Minh Hòa, Nguyễn Ngọc Lâu, 2013):

$$
\begin{gathered}
M_{x y}= \pm \frac{1}{\sqrt{2}} \cdot\left(\frac{M_{S}}{1 \mathrm{~cm}}\right) \cdot 10^{-9} \cdot b, \\
m_{\bar{H}}= \pm \frac{M_{S}}{\sqrt{3}} \cdot \sec B,
\end{gathered}
$$

ở đây $M_{S}$ - độ chính xác của lịch vệ tinh $(\mathrm{cm}) ; \mathrm{b}$ - chiều dài vector baseline giữa hai điểm.

Với chiều dài baseline $\mathrm{b}=100 \mathrm{~km}$ và sử dụng lịch vệ tinh có độ chính xác $M_{S}=2 \mathrm{~cm}$ chúng ta sẽ nhận được độ chính xác vị trí mặt bằng tương hỗ $M_{x y}=0,2 \mathrm{~cm}$ và độ chính xác của độ cao trắc

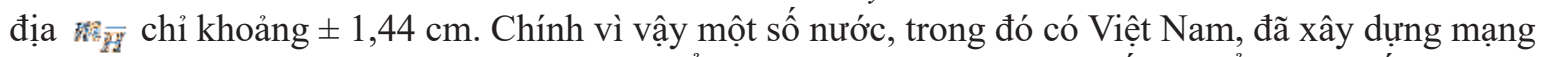
lưới GNSS thưa với mật độ 70 - $120 \mathrm{~km} / 1$ điểm được gọi là “mạng lưới cấp 0" để giải quyết bài toán định vị ellipsoid các định điểm tọa độ gốc và nâng cao độ chính xác của mạng lưới thiên văn - trắc địa hạng I, II.

Mạng lưới thiên văn - trắc địa hạng I, II được xử lý toán học bằng cách như vậy cũng có hạn chế chế. Bản chất hệ tọa độ phẳng quốc gia cũng chỉ là hệ tọa độ $2 \mathrm{D}$. Do đó khí xác định 07 tham số chuyển các tọa độ từ ITRF về hệ tọa độ quốc gia, thực chất chúng ta chỉ thực hiện chuyển chính xác các tọa độ trắc địa ${ }_{x} x_{2}$, của điểm từ WGS84 quốc tế thành các tọa độ trắc địa $B, L$ trong hệ tọa độ $2 \mathrm{D}$ quốc gia. Còn các độ cao trắc địa của các điểm sẽ có sai số rất lớn và không đảm bảo để xác định các độ cao quasigeoid độ chính xác cao trên lãnh thổ Việt Nam. Như đã phân tích trong (Hà Minh Hòa, 2019b), dựa theo (Hướng dẫn sử dụng các tham số tính chuyển từ Hệ tọa độ quốc tế WGS-84 sang Hệ tọa độ quốc gia VN-2000 và ngược lại) các tham số chuyển các tọa độ từ Hệ tọa độ quốc tế WGS-84 sang Hệ tọa độ quốc gia VN-2000 bằng:

$$
\begin{aligned}
& d X_{0}=191,90441429 m, d Y_{0}=39,30318279 m, d Z_{0}=111,45032835 \mathrm{~m}, \\
& \varepsilon_{X}=0^{\prime \prime}, 00928836, \varepsilon_{Y}=-0^{\prime \prime}, 01975479, \varepsilon_{Z}=0^{\prime \prime}, 00427372, \\
& \Delta m=-0,000000252906278 .
\end{aligned}
$$

Khi chuyển độ cao trắc địa từ WGS84 vê hệ tọa độ VN2000, chúng ta sử dụng công thức:

$$
H=\bar{H}+\cos \bar{B} \cdot \cos \bar{L} \cdot d X_{0}+\cos \bar{B} \cdot \sin \bar{L} \cdot d Y_{0}+\sin \bar{B} \cdot d Z_{0}+\Delta H\left(\varepsilon_{X}, \varepsilon_{Y}, \varepsilon_{Z}, \Delta m\right),
$$

ở đây thành phần

$$
\Delta H\left(\varepsilon_{X}, \varepsilon_{Y}, \varepsilon_{Z}, \Delta m\right)=\frac{1}{2} \cdot e^{2} \cdot \bar{N} \cdot \sin 2 \bar{B} \cdot\left(\cos \bar{L} \cdot \varepsilon_{Y}-\sin \bar{L} \cdot \varepsilon_{X}\right)+\left(\frac{a^{2}}{\bar{N}}+\bar{H}\right) \cdot \Delta m .
$$


Khi định vị ellipsoid với điều kiện các trục tọa độ của WGS84 song song với các trục tọa độ tương ứng của VN2000. Do đó về lý thuyết, đại lượng $\Delta H\left(\varepsilon_{X}, \varepsilon_{Y}, \varepsilon_{Z}, \Delta m\right)$ phải bằng 0 . Tuy nhiên do độ thay đổi tỷ lệ xích $\Delta m=-0,000000252906278$ nên độ cao trăc địa $H$ sẽ chứa thêm sai số ở

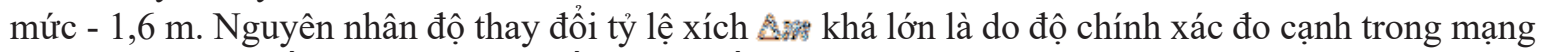
lưới thiên văn - trắc địa hạng I, II thấp hơn nhiều so với công nghệ GNSS.

Vào những năm đầu của nhưng năm 1990, trong quá trình xử lý dữ liệu GNSS người ta đã tính đến các hiệu ứng địa động lực diễn ra trên bề mặt trái đất như các hiệu ứng triều trái đất, triều cực trái đất, sức tải của khí quyển và sức tải của đại dương (Hà Minh Hòa, Nguyễn Ngọc Lâu, 201). Ngoài ra, do sự chuyển dịch của tâm vật chất trái đất với tốc độ $1 \mathrm{~mm} / 1$ năm làm các tọa độ không gian của các điểm trong ITRF bị thay đổi, nên khi xử lý các dữ liệu GNSS trong ITRF các tọa độ không gian của các điểm IGS được sử dụng sẽ được chuyển về thời điểm $\mathrm{t}_{0}$ mà tại thời điểm đó ITRF đã được định vị vào tâm vật chất trái đất theo công thức:

$$
\chi(t)=\chi\left(t_{0}\right)+T+D \cdot \chi\left(t_{0}\right)+R \cdot \chi\left(t_{0}\right),
$$

ở đây các tham số chuyển đổi tọa độ $T, D$ và ma trận $R$ được xác định vào thời điểm to thêm vào đó ma trận $R$ có dạng:

$$
R=\left(\begin{array}{ccc}
0 & -R_{3} & R_{2} \\
R_{3} & 0 & -R_{1} \\
-R_{2} & R_{1} & 0
\end{array}\right),
$$

các góc xoay Euler $R_{1}=-\varepsilon_{X}^{t}, R_{2}=-\varepsilon_{Y}^{t}, R_{3}=-\varepsilon_{Z}^{t}, R_{1}=\varepsilon_{X}^{t}, R_{2}=\varepsilon_{Y}^{t}, R_{3}=\varepsilon_{Z}^{t}$ : độ thay đổi tỷ lệ

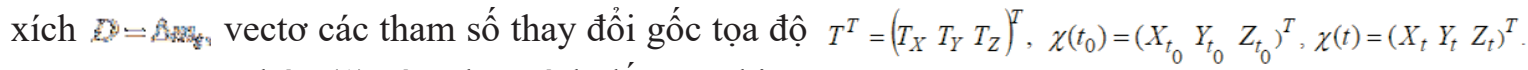

Trong công thức (1) còn chưa tính đến một hiện tượng địa động lực - đó là chuyên động mảng. Vấn đề này sẽ được xem xét trong mục 2 dưới đây.

Hệ tọa độ không gian quốc gia được xây dựng dựa trên ITRF khi tính đến các hiệu ứng địa động lực nêu trên được gọi là hệ tọa độ động lực quốc gia. Ý tưởng phát triển hệ tọa độ động lực quốc gia ở Việt Nam đã được đề xuất trong (Hà Minh Hòa, Đặng Hùng Võ, Phạm Hoàng Lân, Nguyễn Ngọc Lâu và nnk, 2005) nhằm mục đích xây dựng hệ tọa độ không gian quốc gia dựa trên ITRF nhằm các mục đích:

- Thống nhất các mạng lưới thiên văn - trắc địa hạng I, II và mặt lưới độ cao quốc gia hạng I, II thành mạng lưới không chế tọa độ không gian thống nhất dựa trên công nghệ GNSS;

- Nâng cao độ chính xác của các độ cao trắc địa để vừa đảm bảo yêu cầu của hệ tọa độ $3 \mathrm{D}$, vừa đáp ứng yêu cầu xác định các giá trị độ cao quasigeoid theo phương pháp GNSS/thủy chuẩn.

- Đảm bảo khai thác các mô hình toàn cầu như EGM, MDT để giải quyết các bài toán trắc địa vật lý trên lãnh thổ quốc gia.

Việc xây dựng hệ tọa độ động lực quốc gia ở Việt Nam đã được phản ánh trong Chiến lược phát triển ngành Đo đạc và Bản đồ đến năm 2020 (Quyết định số 33/2008/QĐ-TTg). Trong (Hà Minh Hòa, 2019) chúng ta đã đưa ra tiêu chí để xác định các điểm khống chế tọa độ không gian quốc gia trong hệ tọa độ không gian quốc gia và mật độ của chúng. Trong bài báo khoa học này chúng ta sẽ xem xét vấn đề khai thác hệ độ cao quốc gia trong việc xây dựng hệ tọa độ không gian quốc gia và xu hướng xây dựng hệ tọa độ không gian quốc gia gắn liền với mảng kiến tạo. 


\section{Giải quyết vấn đề}

Trong (Quy phạm xây dựng lưới độ cao Nhà nước hạng 1,2,3 và 4 (1988)) đã chỉ rõ rằng hệ độ cao quốc gia Việt Nam là hệ độ cao chuẩn với mặt khởi tính là mặt nước biển trung bình nhiều năm tại trạm nghiệm triều Hòn Dấu. Do các tính chất trên đã phản ánh trong cơ sở dữ liệu (CSDL) độ cao quốc gia và các tư liệu bản đồ địa hình quốc gia, nên không có lý do gì để Việt Nam quay sang sử dụng mô hình geoid mà bỏ đi mô hình quasigeoid quốc gia. Điều này có nghĩa là Việt Nam phải xây dựng mô hình quasigeoid quốc gia, chứ không phải mô hình geoid quốc gia.

Một trong những cơ sở quan trọng để xây dựng hệ tọa độ không gian quốc gia là xây dựng mô hình quasigeoid quốc gia độ chính xác cao. Điều này đã được luận giải chi tiết trong (Hà Minh Hòa, và nnk, 2012). Một mặt mô hình quasigeoid quốc gia độ chính xác cao cho phép xác định độ cao trắc địa độ quốc gia chính xác cao dựa trên các độ cao chuẩn hạng $\mathrm{I}, \mathrm{II}$ - một thành phần trong các tọa độ trắc địa $\mathrm{B}, \mathrm{L}, \mathrm{H}$ của các điểm. Mặt khác, nó cho phép xác định các các độ cao chuẩn hang $\mathrm{I}$, II dựa trên công nghệ GNSS. Độ cao trắc địa quốc gia của một điểm là độ cao của điểm đó so với mặt ellipsoid quy chiếu quốc gia.

Để đảm bảo yêu cầu các định các tọa độ không gian của một điểm trong hệ tọa độ không gian quốc gia với sai số tương đối ở mức tức tương đương với độ chính xác tương đổi của các tọa độ không gian của điểm đó trong ITRF, độ chính xác của mô hình quasigeoid quốc gia phải cao hơn 4 cm (Hà Minh Hòa, 2014a).

Các dữ liệu được sử dụng để xây dựng mô hình quasigeoid quốc gia độ chính xác cao là mô hình trọng trường toàn cầu EGM, mạng lưới độ cao hạng I, II quốc gia và các dữ liệu trọng lực.

Như đã trình bày trong (Hà Minh Hòa, và nnk, 2012), do đã khai thác trong một thời gian dài, mạng lưới độ cao hạng I, II quốc gia ở nhiều nước đòi hỏi phải đo lại do nhiều mốc độ cao hoặc bị mất trên thực địa, hoặc bị xê dịch do các tác nhân nhân sinh và tự nhiên. Tuy nhiên điều đó đòi hỏi kinh phí khá lớn và mất hàng thập kỷ và không đáp ứng được yêu cầu của hiện tại. Ví dụ ở Canada hệ độ cao quốc gia chính thức được sử dụng ở Canada vào năm 1935 với khoảng 70.000 mốc thủy chuẩn và chủ yếu nằm dọc các quốc lộ, các tuyến đường sắt ở Nam Canada. Đối với mạng lưới độ cao quốc gia này, nhiều mốc thủy chuẩn đó bị mất và chi phí bảo quản các mốc thủy chuẩn này rất cao. Việc hiện đại mạng lưới độ cao này đòi hỏi kinh phí khổng lồ và phải mất hàng chục năm mới hoàn thành (Height Reference System Modernization; Veronneau M., Huang J., 2011). Do vậy Canada đã quyết định loại bỏ hệ độ cao quốc gia CGVD 1928 cũ và sử dụng các mô hình trọng trường toàn cầu EGM, dữ liệu trọng lực để xây dựng mô hình geoid với tên gọi là CGG2013 mới được công bố vào tháng 11/2013. Độ chính xác của mô hình geoid CGG2013 cao hơn $\pm 3 \mathrm{~cm}$ trên 80\% phần lục địa của Canada. Các độ cao chính trong hệ độ cao mới CGVD2013 được xác định dựa trên các kết quả đo GNSS từ 250 trạm thuộc hệ thống kiểm soát tích cực Canada (CACS), mạng lưới cơ sở Canada (CBN) và mô hình geoid CGG2013 (Véronneau M., 2014).

Ở Vương quốc Anh từ mạng lưới độ cao quốc gia cũ chỉ tìm được 179 điểm độ cao ổn định trong mạng lưới độ cao quốc gia. Mô hình geoid trọng lực OSGM02 độ phân giải $1.5 \mathrm{~km}$ x $1.5 \mathrm{~km}$ của Vương quốc Anh được xây dựng dựa trên các dữ liệu đo trọng lực trên đất liền và trên biển của $\mathrm{Co}$ quan Khảo sát địa chất (BGS) của Anh, mô hình EGM96 của Mỹ, mô hình trọng lực KMS98 được xác định từ các dữ liệu altimetry của Đan Mạch, các dữ liệu trọng lực của Cục trọng lực quốc tế (BGI) của Pháp và mô hình số độ cao độ phân giải $50 \mathrm{~m} \times 50 \mathrm{~m}$ của Anh. 179 điểm độ cao ổn định được sử dụng để làm khớp với mô hình geoid trong lực $\mathrm{OSGM} 02$ nhằm nâng cao độ chính xác của mô hình này (Forsberg R., Strykowski G., Iliffe J.C., Ziebart M., Cross P.A., Tscherning C.C., 
Cruddace P., 2001; Iliffe J.C., Ziebart M., Cross P.A., Forsberg R., Strykowski G., Tscherning C.C., 2003).

Ở Thổ Nhĩ Kỳ chỉ tìm thấy 87 điểm độ cao ổn định trong mạng lưới độ cao quốc gia. Các điểm độ cao ổn định này được sử dụng để làm khớp với mô hình quasigeoid toàn cầu EGM2008 để xây dựng mộ hình quasigeoid quốc gia (Metin Soycan, 2014).

Như vậy với mục tiêu đảm bảo cơ sở không chế độ cao quốc gia đáp ứng yêu cầu cung cấp nhanh chóng các tư liệu bản đồ địa hình cho các hoạt động kinh tế - xã hội, trong trường hợp nhiều mốc độ cao bị mất hoặc bị xê dịch, nhiều quốc gia đã sử dụng các mốc độ cao còn ổn định trên thực địa kết hợp với các mô hình trọng trường toàn cầu EGM và dữ liệu trọng lực để xây dựng mô hình geoid/quasigeoid quốc gia độ chính xác cao.

Cũng có một đặc điểm nữa là các nước không chờ xây dựng xong mô hình geoid/quasigeoid độ chính xác cao mới xây dựng hệ quy chiếu không gian quốc gia. Ví dụ ở Australia, mặc dù mô hình quasigeoid quốc gia AUSGeoid98 mới đạt độ chính xác \pm 0,4 m (Sussanna, V., V. Janssen, and P. Gibbings, 2014) đã xây dựng hệ quy chiếu không gian quốc gia GDA1998. Như vậy hệ xây dựng hệ quy không gian quốc gia ở các nước được xây dựng theo từng giai đoạn: sau mỗi lần xây dựng được mô hình geoid/quasigeoid với độ chính xác cao hơn, người ta lại xây dựng hệ quy không gian quốc gia mới và quá trình tiệm tiến như vậy cứ tiếp tục. Ví dụ nước Mỹ xây dựng mô hình geoid USGG09 với độ chính xác $\pm 3-4 \mathrm{~cm}$ tương ứng với hệ quy không gian quốc gia NAD83. Nước Mỹ sẽ xây dựng hệ quy không gian quốc gia NSRS2022 tương ứng mô hình geoid GEOID2022 với độ chính xác $\pm 1-2 \mathrm{~cm}$ (Roman, D. R., Y.M. Wang, J. Saleh, X. Li, W. Waickman).

Ở Việt Nam trong (Ha Minh Hoa, 2017; Ha Minh Hoa, 2018a) đã trình bày mô hình quasigeoid quốc gia VIGAC2017 với độ chính xác ở mức $\pm 5,8 \mathrm{~cm}$ và hệ quy chiếu không gian quốc gia khởi đầu VIGAC2017.

Vấn đề cuối cùng liên quan đến câu hỏi: Hệ quy chiếu không gian quốc gia là hệ tọa độ động lực gắn liền với ITRF hay là hệ tọa độ không gian gắn liền với mảng kiến tạo?

Hệ tọa độ không gian gắn liền với mảng kiến tạo thực chất là hệ tọa độ không gian được cố định với mảng kiến tạo, nhưng chuyển động quán tính so với ITRF. Nó chính là hệ tọa độ Tisserand.

Từ năm 2012 tổ chức IGS đã đưa ra dịch vụ mới: 07 tham số $\mathscr{F}_{z} D_{y}$ R để tính các số hiệu chỉnh vào các tọa độ do chuyển động mảng được xác định vào thời điểm $t_{0}$ (Altamimi, Z., Métivier, L., and Collilieux, X., 2012).Khi đó, thay cho công thức (1) chúng ta sử dụng công thức:

$$
\chi(t)=\chi\left(t_{0}\right)+T+D \cdot \chi\left(t_{0}\right)+R \cdot \chi\left(t_{0}\right)+\dot{\chi}\left(t_{0}\right) \cdot\left(t-t_{0}\right),
$$

ở đây thành phần $\mathscr{K}\left(\varepsilon_{0}\right)$ là số hiệu chỉnh tọa độ không gian do chuyển dịch của mảng và được tính theo công thức (Altamimi, Z., Métivier, L., and Collilieux, X., 2012):

$$
\dot{\chi}\left(t_{0}\right)=\dot{T}+\dot{D} \cdot \chi\left(t_{0}\right)+\dot{R} \cdot \chi\left(t_{0}\right),
$$

ở đây ma trận

$$
\dot{R}=\left(\begin{array}{ccc}
0 & -\dot{R}_{3} & \dot{R}_{2} \\
\dot{R}_{3} & 0 & -\dot{R}_{1} \\
-\dot{R}_{2} & \dot{R}_{1} & 0
\end{array}\right) .
$$


Theo tài liệu (Argus, D.F., R.G. Gordon, M.B. Heflin, C. Ma, R.J. Eanes, P. Willis, W.R. Peltier, and S.E. Owen, 2010), thành phần $\mathscr{X}_{\left(\hat{\theta}_{0}\right)}$ có thể được tính theo công thức sau:

$$
\dot{\chi}\left(t_{0}\right)=\dot{T}+\dot{D} \cdot \chi\left(t_{0}\right)+\left\lfloor\omega_{p} \otimes\right\rfloor \chi\left(t_{0}\right),
$$

ở đây ma trận

$$
\left[\omega_{p} \otimes\right]=\left(\begin{array}{ccc}
0 & -\Omega_{Z} & \Omega_{Y} \\
\Omega_{Z} & 0 & -\Omega_{X} \\
-\Omega_{Y} & \Omega_{X} & 0
\end{array}\right),
$$

các tốc độ góc Euler $\Omega_{X}, \Omega_{Y}, \Omega_{Z}$ của mảng kiến tạo.

Hệ quy chiếu không gian quốc gia và các công thức hiệu chỉnh các tọa độ do chuyển dịch mảng được trình bày chi tiết trong các chương 15, 17 của tài liệu (Hà Minh Hòa, 2018b).

Hệ quy chiếu không gian quốc gia (NSRS) ở Mỹ là hệ tọa độ ba chiều sẽ hoàn thành vào năm 2022 và bao gồm 04 khung quy chiếu Trái đất: NATRF2022, PTRF2022, CTRF2022, MTRF2022. Theo tài liệu (Roman, D., 2017), bốn khung quy chiếu nêu trên, còn được gọi là các khung quy chiếu được gắn liền với mảng kiến tạo, lần lượt nằm trên các mảng kiến tạo Bắc Mỹ (North America NA), Thái Bình Dương (Pacific - P), Caribbe (C), Mariana (M) tương ứng với khung quy chiếu không gian quốc gia NSRF2022 - là sự triển khai NSRS của nước Mỹ, thêm vào đó NRSF2022 liên kết chặt chẽ với ITRF2014 vào thời điểm chuẩn 2010.0.

Như vậy, với cách tiếp cận việc xây dựng hệ quy chiếu không gian quốc gia gắn liền với mảng kiến tạo như đã trình bày ở trên, chúng ta sẽ đạt được các lợi ích sau:

- Các tọa độ không gian của các điểm trắc địa nằm trên mảng kiến tạo sẽ không thay đổi theo thời gian trong hệ quy chiếu không gian quốc gia do chuyển dịch của mảng kiến tạo. Điều này đảm bảo sự ổn định của các hệ quy chiếu tọa độ mặt phẳng và độ cao quốc gia;

- Dựa trên các kết quả đo lặp mạng lưới GNSS được xây dựng trên mảng kiến tạo có các tọa độ không gian cả trong hệ quy chiếu không gian quốc gia và lẫn trong ITRF, chúng ta có dữ liệu không gian rất quan trọng để hiệu chỉnh thường xuyên các vận tốc góc Euler của mảng kiến tạo này nhằm đóng góp vào việc hoàn thiện mô hình động lực mảng kiến tạo, xác định sự biến dạng của lãnh thổ quốc gia do các hoạt động nội sinh bên trong vỏ Trái đất thuộc lãnh thổ quốc gia, phối hợp với tổ chức IGS để hiệu chỉnh thường xuyên ITRF vào các thời điểm khác nhau;

- Trong trường hợp các tọa độ không gian của các điểm GNSS thuộc mạng lưới GNSS địa động lực quốc gia đã được xác định trong hệ quy chiếu không gian quốc gia gắn liền với mảng kiến tạo, nếu phát hiện thấy sự dịch chuyển của các điểm GNSS, thì yếu tố chuyển động mảng kiến tạo bị loại 
trừ và nguyên nhân là do các hoạt động địa chất nội sinh bên trong lớp vỏ Trái đất thuộc lãnh thổ quốc gia gây ra, ví dụ hoạt động của các đới đứt gãy thuộc mảng kiến tạo, sự sụt lún của vùng lãnh thổ v..v. Khi loại trừ ảnh hưởng của sự chuyển động của mảng kiến tạo, đây là tiền đề để nghiên cứu sự biến dạng lãnh thổ quốc gia do các hoạt động địa chất nội sinh khác gây ra.

\section{Kết luận}

Quá trình xây dựng hệ quy chiếu không gian quốc gia gắn liền với việc xây dựng mô hình quasigeoid độ chính xác cao trên lãnh thổ Việt Nam. Về phần mình, do chưa hoàn thành việc đo đạc trọng lực chi tiết trên lãnh thổ quốc gia, nên việc sử dụng mạng lưới độ cao hạng I,II để xây dựng mô hình quasigeoid độ chính xác cao có ý nghĩa đặc biệt. Tuy nhiên để hoàn thiện mạng lưới độ cao hạng I,II ở Việt Nam đòi hỏi chi phí nhiều kinh phí và tốn thời gian đến hàng thập kỷ. Để đáp ứng kịp thời việc đảm bảo dữ liệu phục vụ các nhu cầu phát triển kinh tế - xã hội của đất nước, từ kinh nghiệm quốc tế chúng ta có thể từng bước xây dựng mô hình quasigeoid quốc gia dựa trên các mô hình trọng trường trái đất hiện có và các độ cao trên các mốc độ cao hạng I, II ổn định và song song với công việc đó tiến hành xây dựng hệ quy chiếu không gian quốc gia. Quá trình này có thể lặp cho đến khi nhận được mô hình quasigeoid quốc gia độ chính xác cao cùng với hệ quy chiếu không gian quốc gia tương ứng.

Tất yếu hệ quy chiếu không gian quốc gia sẽ là hệ quy chiếu không gian gắn liền với mảng Á Âu ở Việt Nam. O

\section{Tài liệu tham khảo}

[1]. Altamimi, Z., Métivier, L., and Collilieux, X., 2012. ITRF2008 plate motion model. Journal of Geophysical research, Vo. 117, pp. 1 - 14, B07402, doi: 10.1029/2011JB008930.

[2]. Argus, D.F., R.G. Gordon, M.B. Heflin, C. Ma, R.J. Eanes, P. Willis, W.R. Peltier, and S.E. Owen, 2010. The angular velocities of the plates and the velocity of Earth's centre from space geodesy, Geophys. J. Int., 180 (3), 916 - 960, doi: 10.1111/j.1365 - 246X. 2009.04463.x.

[3]. Forsberg R., Strykowski G., Iliffe J.C., Ziebart M., Cross P.A., Tscherning C.C., Cruddace P., 2001. OSGM02: A new geoid model of the British Isles. www.discovery.ucl.ac.uk> ... UCL.DISCOVERY

[4]. Hà Minh Hòa, Đặng Hùng Võ, Phạm Hoàng Lân, Nguyễn Ngọc Lâu và nnk, 2005. Nghiên cứu cơ sở khoa học của việc xây dựng mạng lưới GPS các cấp hạng trong Hệ tọa độ động học. Báo cáo Tổng kết khoa học và kỹ thuật đề tài nghiên cứu khoa học công nghệ cấp Bộ Tài nguyên và Môi trường giai đoạn 2002 - 2004. Bộ Tài nguyên và Môi trường. Hà Nội - 2005.

[5]. Hà Minh Hòa, và nnk, 2012. Nghiên cứu cơ sở khoa học của việc hoàn thiện hệ độ cao gắn liền với việc xây dựng hệ tọa độ động lực quốc gia, 247 trg.. Đề tài khoa học và công nghệ cấp Bộ Tài nguyên và Môi trường giai đoạn 2010 - 2012. Hà Nội - 2012. 
[6]. Hà Minh Hòa, Nguyễn Ngọc Lâu, 2013. Nguyên lý lý thuyết và thực tiễn của Trắc địa vũ trụ. Nhà Xuất bản Khoa học và Kỹ thuật, 276 trg., Hà Nội - 2013. ISBN: 893 - 504 - 89 - 3181 - 3.

[7]. Hà Minh Hòa, 2014a. Phương pháp xử lý toán học các mạng lưới trắc địa quốc gia. Nhà Xuất bản Khoa học và Kỹ thuật, 244 trg., Hà Nội - 2014. ISBN: 978-604-67- 0301-3.

[8]. Hà Minh Hòa, 2014b. Lý thuyết và thực tiễn của Trọng lực trắc địa. Nhà Xuất bản Khoa học và Kỹ thuật, 592 trg., Hà Nội - 2014. ISBN: 978-604-67- 0301-3.

[9]. Ha Minh Hoa, 2017. Construction of inital national quasigeoid model VIGAC2017, First step to national spatial reference system in Vietnam. Vietnam journal of Earth Sciences, 39 (2): 155 166 ,

DOI: 10.15625/0866-7187/39/2/9702, Vietnam Academy of Science and Technology, http://www.vjs.ac.vn/index.php/jse.

[10]. Ha Minh Hoa, 2018a. Improvement of the accuracy of the quasigeoid model VIGAC2017. Vietnam journal of Earth Sciences, 40 (1): 38 - 45, DOI: 10.15625/0866-7187/40/1/10914, Vietnam Academy of Science and Technology, http://www.vjs.ac.vn/index.php/jse

[11]. Hà Minh Hòa, 2018b. Một số vấn đề hiện đại của Trắc địa vật lý. Nhà Xuất bản Khoa học và Kỹ thuật, 964 trg., ISBN: 978 - 604 - 67 - 1116 - 2, Hà Nội - 2018.

[12]. Hà Minh Hòa, 2019. Nâng cao độ chính xác của mô hình quasigeoid quốc gia nhờ xây dựng hệ quy chiếu không gian quốc gia. Tạp chí Khoa học Đo đạc và Bản đồ, Số 40, trg. 1-10, tháng Ba năm 2019, ISSN: 0866- 7705, Viện Khoa học Đo đạc và Bản đồ.

[13]. Height Reference System Modernization. National Resources Canada. www.nrcan.gc.ca.

[14]. Hướng dẫn sử dụng các tham số tính chuyển từ Hệ tọa độ quốc tế WGS-84 sang Hệ tọa độ quốc gia VN-2000 và ngược lại. Công văn số 1123/ĐĐBĐ-CNTĐ ngày 26/10/2007 của Cục Đo đạc và Bản đồ Việt Nam.

[15]. Iliffe J.C., Ziebart M., Cross P.A., Forsberg R., Strykowski G., Tscherning C.C., 2003. OSGM02: A New model for converting GPS-derived heights to local height datums in Great Britain and Ireland. Star - Science and Technology of Archelogical Research, Vol. 37, Issue 290, pp. 276 293.

[16]. Metin Soycan, 2014. Improving EGM2008 by GPS and leveling data at local scale, BCG Boletin de Cincias Geodésicas Sec, Artigos, Curitiba, V.20, No.1, PP. 3-18, on - lineversion, ISSN 1982-2170, http://dx.doi.org/10.1590/S1982-21702014000100001.

[17]. Quyết định số 33/2008/QĐ-TTg ngày 27/02/2008 của Thủ tướng Chính phủ về việc phê duyệt Chiến lược phát triển ngành Đo đạc và Bản đồ Việt Nam đến năm 2020.

[18]. Quy phạm xây dựng lưới độ cao Nhà nước hạng 1,2,3 và 4 (1988). Cục Đo đạc và Bản đồ 
Nhà nước. Hà Nội - 1988.

[19]. Roman, D., 2017. The U.S. National Spatial Reference System in 2022 (8841). FIG Working Week 2017: Serveying the world of tomorrow - from digitalisation to augmented reality, Helsinki, Finland, May 29 - June 2, 2017.

[20]. Roman, D. R., Y.M. Wang, J. Saleh, X. Li, W. Waickman, 2009. USGG2009 \& GEOID09: New geoid height models for surveying/GPS. NOAA's National Geodetic Survey, ACSM - MARLS - UCLS - WFPS Conference 2009, 20 February 2009, Salt Lake City, UT.

[21]. Sussanna, V., V. Janssen, and P. Gibbings, 2014. Absolute performance of AUSGeoid09 in mountainous regions. Journal of Applied Geodesy, 8(3), 195-203.

[22]. Veronneau M., Huang J., 2011. A New Gravimetric Geoid Model for Canada. CGG2010, CGU Annual Scientific Meeting, Bauff, Alberta, Canada, 15-18 May.

[23]. Véronneau M., 2014. The Canadian Geodetic Vertical Datum of 2013. Canadian Institute of Geomatics, Ottawa Branch, 29 April 2014, Natural Resources Canada, 33 p. $O$

\section{Summary}

National spatial reference system closed with tectonic plate - tendency of construction of modern national reference system in the world

\section{Ha Minh Hoa}

Vietnam Institute of Geodesy and Cartography

In process of construction of national spatial reference system, an interested problem is stability of the national height system for building of high accurate quasigeoid model. Because the national height system had been exploited a long time, many fist and second orders benchmarks had been displaced from social - economical and tectonical activities, repeat measurement of first and second orders networks requires many cost and time, then many countries only had selected stable fist and second orders benchmarks to fit to the national quasigeoid model constructed by gravimetric data. In Vietnam, quasigeoid model VIGAC2017 had been constructed by this approach. Apart from that at present the national spatial reference system is reference system closed with tectonic plate. This scientific article will consider above mentioned problems. $\bigcirc$ 